\title{
Neurotoxic Lesions of the Dorsomedial Thalamus Impair the Acquisition But Not the Performance of Delayed Matching to Place by Rats: a Deficit in Shifting Response Rules
}

\author{
Peter R. Hunt ${ }^{1}$ and John P. Aggleton ${ }^{2}$ \\ ${ }^{1}$ Medical Research Council Cambridge Centre for Brain Repair, University of Cambridge, Cambridge, CB2 2PY, United \\ Kingdom, and 'School of Psychology, Cardiff University, Cardiff, CF1 3YG, United Kingdom
}

This study examined the acquisition of a T-maze matching to place task by rats with neurotoxic lesions of the thalamic nucleus medialis dorsalis. This test of spatial working memory also entails learning a task rule that is contrary to the animals' innate preference. The rats next performed the same matching task over different retention delays. Finally, they were trained on a reversal of the task rule, i.e., to nonmatch to place. Although the lesions produced a clear acquisition impairment on the matching task, there was no evidence of a loss of working memory. A series of control tasks found no appreciable effect on a conditioned cue preference task or on open field activity. The pattern of results shows that medialis dorsalis lesions lead to a selective increase in perseverative behavior that can retard task acquisition. This perseverative deficit closely resembles that observed after prefrontal damage in rats, strongly indicating dysfunction in a common system.

Key words: medial dorsal thalamus; matching to place; response rules; working memory; spatial memory; prefrontal cortex; amnesia
Although clinical and experimental studies have long implicated the thalamic nucleus medialis dorsalis in learning and memory (Markowitsch, 1982), its contribution remains ill-defined. This is exemplified by the apparent failure of lesions of medialis dorsalis in rats to reveal a consistent pattern of deficits on tests of working memory. One way of adopting a more systematic approach is to consider the possible functions of medialis dorsalis in the light of those brain regions with which it is heavily connected. Foremost among these connections are those with the prefrontal cortex, whose extent has indeed been defined by its connections with medialis dorsalis.

Although it has been supposed that the prefrontal cortex might have a general role in working memory, it is most often thought to be involved in aspects of response or attentional control and their inhibition (Cohen et al., 1996; Robbins, 1996). We, therefore, examined the effects of neurotoxic lesions of nucleus medialis dorsalis on a task that taxes these latter attributes of memory. The task selected was delayed matching to place in a T-maze. In previous experiments, we had found that lesions in this nucleus have little or no effect on delayed nonmatching to place (delayed forced alternation) in a T-maze (Hunt and Aggleton, 1991). The nonmatching variant of this task is readily learned because it takes advantage of the spontaneous spatial alternation behavior displayed by normal rats. In contrast, the matching variant requires the rat to learn the rule opposed to its natural bias, and so is much more difficult to acquire. Because prefrontal lesions in rats appear to have the clearest effects on spatial memory tasks

Received June 9, 1998; revised Sept. 3, 1998; accepted Sept. 10, 1998.

This research was supported by a grant from the Wellcome Trust. We thank Rebecca Dias, Chris Shaw, Eduardo Torres, and Shirley Whiteley for their assistance.

Correspondence should be addressed to P. R. Hunt, Medical Research Council Cambridge Centre for Brain Repair, University of Cambridge, Forvie Site, Robinson Way, Cambridge, CB2 2PY, UK

Copyright (C) 1998 Society for Neuroscience $\quad 0270-6474 / 98 / 1810045-08 \$ 05.00 / 0$ when the rat is required to reverse a learned or spontaneous bias (Kolb et al., 1974; Kolb, 1984), it was predicted that lesions of medialis dorsalis might have similar asymmetric effects on these tasks.

All rats underwent two other behavioral tests; the first was a conditioned cue preference task. This class of task assesses the ability to associate a reinforcer with a specific cue signal, using a classical conditioning paradigm (Van der Kooy, 1985). As a consequence, it can help to determine whether lesions of the nucleus medialis dorsalis (MD) disrupt reward-related processes. This is of value because such a deficit could affect the acquisition and performance of a wide array of tasks (Sahgal, 1993), including the matching to place rule, which is more difficult to acquire than the nonmatching rule. Indeed, there is evidence from one study using a cue preference task (McAlonan et al., 1993) that ibotenic acid medialis dorsalis lesions can impair conditioning. The second test examined open field behavior, because previous experiments had indicated that medialis dorsalis lesions might produce an increase in activity (Hunt and Aggleton, 1998). Because such a change might indirectly affect the performance of other tasks, we also tested whether the present thalamic lesions altered activity and exploration.

\section{MATERIALS AND METHODS}

\section{Subjects}

The subjects were 20 naive male rats of the pigmented Dark Agouti (DA) strain (B \& K Universal Ltd, Hull, UK). The rats were $\sim 12$ weeks old and weighed $210-250 \mathrm{gm}$ at the time of surgery. They were housed individually in a single holding room with a photoperiod of $14 / 10 \mathrm{hr}$ light/dark. Each rat was randomly assigned to one of two surgical groups, MD (lesions of the thalamic nucleus medialis dorsalis) or SHAM (surgical controls). There were ten rats in each group, and each was maintained on $\sim 15 \mathrm{gm}$ of laboratory diet (RM1E; Special Diets Services, Witham, UK) per day. Throughout the testing period body weights were monitored to ensure that rats remained at no less than $85 \%$ of normal weight. Experiments were conducted in accordance with the United Kingdom Animals (Scientific Procedures) Act, 1986. 


\section{Apparatus and procedure}

Conditioned cue preference. The apparatus consisted of a central octagonal arena with eight radial arms. The central arena was $34 \mathrm{~cm}$ in diameter and constructed of a varnished plywood floor with transparent acrylic sheet walls $24 \mathrm{~cm}$ in height. The arms were $86 \mathrm{~cm}$ in length and $10 \mathrm{~cm}$ in width and, like the center, were constructed of a plywood floor and transparent acrylic walls. A food well $(2 \mathrm{~cm}$ in diameter and $0.5 \mathrm{~cm}$ deep) in which reward pellets could be placed was located $2 \mathrm{~cm}$ from the end of each arm. A transparent guillotine door was located at the junction of each arm to the central arena, and these could be raised and lowered either together or independently by a system of overhead cords. The entire maze was set on a circular turntable. This enabled the arms and the central hub to be rotated through $360^{\circ}$. Lighting was provided by three fluorescent lights $140 \mathrm{~cm}$ above the maze.

The guillotine doors to six of the maze arms were closed, leaving access from the central area to only two arms. The side walls, end walls, and top of one of these arms was completely enclosed in black polythene sheeting and the other in white polythene sheeting. This ensured that the two arms were visually highly distinctive. Because the polythene sheeting could be applied to any of the arms it was possible to change the appearance of any arm as required.

All rats were maintained on a restricted feeding regimen for $5 \mathrm{~d}$ before testing and throughout the testing period, their body weights were not allowed to fall below $85 \%$ of normal. On the first day of testing (session 1 ), each rat was put into the center of the maze and allowed free access for $20 \mathrm{~min}$ to the two open arms, enclosed in black and white polythene as described above and located opposite each other. No food was present in the maze during this session. Time spent in each of the two arms, and the number of entries to each arm were recorded by the experimenter, who sat in the same location, $1.5 \mathrm{~m}$ from the apparatus, throughout all test sessions.

On test days 2-9, each rat was randomly assigned two of the eight arms of the maze, one black and one white. These arms always had at least two closed arms between them. The pattern of arm assignment for individual rats in the SHAM group replicated that for rats in the MD group. Half of the rats from each group were enclosed by a wooden block in the "baited" arm and half in the "nonbaited" arm for a period of $20 \mathrm{~min}$ on each test session. Twenty grams of the subjects' normal laboratory rat diet (RM1E; Special Diets Services) was scattered around the floor of the baited arms, whereas the nonbaited arms contained no food. The selection of baited and nonbaited was counterbalanced between black and white arms. The confinement to baited and nonbaited arms was alternated for each session so that each rat received equal exposure to baited and nonbaited arms. Before each day's testing, the maze was rotated clockwise by one arm, i.e., by $45^{\circ}$, and the polythene covers were moved back by one arm into their former position. Each rat thus remained in the same spatial location but in a different arm to prevent the accumulation of olfactory cues in the arms. The procedure for session 1 was repeated on the tenth and final testing day, i.e., the rat was placed for $20 \mathrm{~min}$ in the apparatus with free access to both arms, neither of which was baited.

Exploration of an open arena ("open field"). The apparatus consisted of a circular arena $90 \mathrm{~cm}$ in diameter with a $45 \mathrm{~cm}$ high wall. The floor and wall of the arena were painted matte black, and the floor was marked with two concentric circles 30 and $60 \mathrm{~cm}$ in diameter. Each of the outer two rings thus formed was divided by radial lines, the outer ring was divided into eight sectors, and the middle ring was divided into four sectors. The center circle was undivided. Illumination of the arena was provided by a fluorescent room light $2 \mathrm{~m}$ above it. A video camera was supported by a tripod $1.75 \mathrm{~m}$ above the arena so that the activity sessions could be recorded without the presence of the experimenter in the room.

Each test session consisted of a rat being placed in an outer sector and allowed to wander about the arena freely for $5 \mathrm{~min}$. When all rats had completed one such session, activity was analyzed from the video recording. All line crossings in which all four paws crossed a line were counted and, of these, inward line crossings were noted. Also, time spent in the two inner rings, i.e., away from the arena wall, was recorded. The experimenter was unaware of the rats' groups during this process.

Matching to place in a T-maze. Apparatus: the T-maze had an aluminum floor $10 \mathrm{~cm}$ wide and clear acrylic sheet walls $17 \mathrm{~cm}$ high. The stem of the maze was $80 \mathrm{~cm}$ long with an aluminum guillotine door $33 \mathrm{~cm}$ from the beginning. The cross arm was $136 \mathrm{~cm}$ long with a food well $4 \mathrm{~cm}$ in diameter and $0.75 \mathrm{~cm}$ deep, located in the floor $2 \mathrm{~cm}$ from each end. The maze was supported on stands $93 \mathrm{~cm}$ high and was illuminated by fluorescent room lights suspended $92 \mathrm{~cm}$ above the apparatus. The luminance at the choice point and food wells was 320 and 280 lux, respectively. Testing was performed in a different room from previous testing but that contained salient visual cues.

Four weeks after the conclusion of the open field test all rats were trained on the acquisition of a matching to place task in a T-maze. Pretraining, which consisted of one habituation session, began 4 weeks after the open field test. This habituation session was immediately followed by task acquisition. For this, each trial consisted of two stages; an "information" run and a "test" run. At the beginning of each trial, the experimenter placed three reward pellets $(45 \mathrm{mg})$ in one food well and closed off the other arm of the maze with a wooden block adjacent to the choice point. The rat was then placed at the start point, and the guillotine door was raised, so allowing the rat to run to the choice point. On this information run, the rat was forced by the wooden block to enter a predetermined arm, where it was allowed to eat all three pellets. The rat was then picked up and returned to the start box. While the rat was retained in the start box, the experimenter baited the arm just visited by the rat with three reward pellets and also went through the motions of baiting the other arm without actually leaving any reward pellets in the food well. The experimenter then removed the wooden block and raised the guillotine door to allow the rat to run to the choice point for a second time (the test run). The delay between the end of the information run and the beginning of the test run was $\sim 10 \mathrm{sec}$. On the test run both arms were open, and the rat was allowed a free choice. The rat was deemed to have made a choice when all four of its paws were in one arm. At this point the wooden block was placed behind it to prevent the rat changing its selection. If a correct choice was made, i.e., the rat entered the same arm as on the information run, the rat was allowed to eat the reward pellets before being returned to the start box for trial two. If an incorrect choice was made the rat was confined to the arm without food reward for $10 \mathrm{sec}$ before being returned to the start box. Each daily session consisted of six trials, and rats were tested in groups of three or four with each rat having one trial in turn. This spaced method meant that there was an intertrial interval of 3-5 min.

When each rat had reached an acquisition criterion of 25 correct trials over five consecutive sessions ( 30 trials), it moved on to the next stage in which three delay conditions of 10,20 , and $40 \mathrm{sec}$ were interposed between the information and test runs. Two trials at each condition were given each day in a pseudorandom order, and each rat received 10 such test sessions followed by three sessions (i.e., 18 trials) of undelayed matching to sample.

The next stage began on the next test session and consisted of a reversal to nonmatching to sample, i.e., rats were now rewarded for selecting in the choice phase the arm opposite to that entered in the sample phase. All other aspects of testing were identical to those used in the initial acquisition of the matching task. Testing continued until each rat had achieved the same acquisition criterion as in the matching to place stage ( 25 of 30 trials).

Surgical procedure. Each rat was deeply anesthetized by intraperitoneal injections of pentobarbitone sodium (Sagatal; Rhone Merieux) at a dose rate of $6 \mathrm{mg} / 100 \mathrm{gm}$. It was then placed in a stereotaxic headholder (David Kopf Instruments, Tujunga, CA), the scalp was retracted, and a small craniotomy was made to expose the dura above the target region. A single injection of $0.36 \mu$ l of a $0.12 \mathrm{M}$ solution of NMDA (Sigma, Poole, UK) dissolved in phosphate buffer, $\mathrm{pH} 7.2$, was made through a $1 \mu \mathrm{l}$ microsyringe (Hamilton Instruments, Bonaduz, Switzerland) in each hemisphere. Each injection was made over a period of $5 \mathrm{~min}$, and the needle was left in position for a further $5 \mathrm{~min}$ before being retracted. The injection coordinates relative to ear bar zero with the incisor bar set at +5.0 were: anteroposterior, 3.7 ; height, 4.6 ; lateral, \pm 0.7 . After removal of the needle from the second hemisphere, the skin was sutured, and wound powder (Acramide, Dales Pharmaceuticals, Skipton, UK) was applied to the area.

A heated pad was kept under the rats at all times during surgery to maintain normal body temperature, and a $6 \mathrm{ml}$ subcutaneous injection of isotonic saline (Animalcare Ltd, York, UK) was made at the beginning of each surgery to prevent dehydration. The eyes were protected from both dehydration and excessive light by the application of ophthalmic ointment (Chloromycetin; Parke-Davis, Pontypool, UK). Immediately after surgery, a further $10 \mathrm{ml}$ of saline was injected along with etamphylline (Millophyline; Arnold's, Romford, UK; $35 \mathrm{mg} / \mathrm{kg}$, s.c.). The surgical procedure for the SHAM rats was identical to that described for the MD rats except that the needle of the Hamilton syringe was lowered to a height of 5.1 above ear-bar 0 and then retracted without making an injection.

Histological procedure. At the end of the study each rat was perfused 

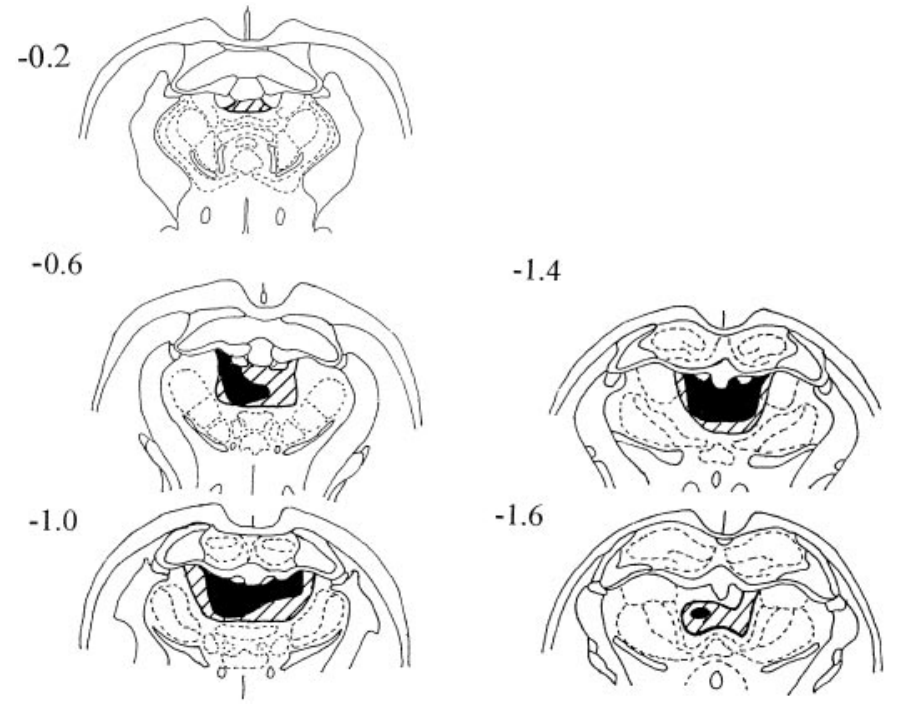

Figure 1. Diagrammatic reconstruction of the lesions in nucleus medialis dorsalis. The coronal sections depict the smallest (black) and largest (diagonal lines) extent of cell loss. The numbers refer to the approximate corresponding anteroposterior levels from the stereotaxic atlas of Pellegrino and Cushman (1967).

intracardially with $0.9 \%$ saline followed by $5 \%$ formol saline. The brains were subsequently blocked, embedded in wax (Paraplast), and cut in 10 $\mu \mathrm{m}$ coronal sections. Every tenth section was mounted and stained with a Nissl stain (cresyl violet).

\section{Statistical analysis}

Where appropriate, parametric tests were used to compare the scores of the two groups. All $t$ tests were one-tailed unless otherwise stated. Analyses of simple effects after ANOVA were based on just the level of the within-subject variable at which the effect was being tested (Keppel, 1973). Error bars on figures refer to SEM.

\section{RESULTS}

\section{Histological analysis}

Damage within $M D$

All ten rats in the MD group had extensive lesions within nucleus medialis dorsalis. The largest and smallest of the MD lesions are depicted in Figure 1. In most cases the lesion affected at least 80\% of the nucleus; the only sparing occurring at the most lateral and ventral limits of the nucleus. The region composing nucleus medialis dorsalis was always shrunken, and within the extent of the lesion there was almost a complete loss of neurons (Fig. 2) but no evidence of either gliosis or infarction.

\section{Damage to other structures}

Loss of cells in the medial portion of nucleus lateralis dorsalis occurred in two cases, one unilateral and one bilateral. Modest damage to the anterior dorsal nucleus was noted in five cases bilaterally and in three cases unilaterally. In all cases the other anterior thalamic nuclei were almost completely spared, but there was damage to the midline nucleus paraventricularis and to that part of nucleus parataenialis at the rostral level of medialis dorsalis. In most cases there was also a limited zone of damage restricted to where the injection tract passed through the dentate gyrus. The habenula did not appear to suffer neurotoxin damage. In all SHAM rats the needle tract could be seen entering the hippocampus, dorsal to medialis dorsalis.

\section{Conditioned cue preference}

The results of one rat from the SHAM group were discarded because of severe noise interference during the critical day 10 test session. This left nine rats in the SHAM group and 10 in the MD group for this test. The performance of the two groups can be seen in Figure 3.

\section{Time in arms}

The time that each rat spent in the arm to be paired with food was recorded on session 1 and termed "cued1"; time spent in the nonbaited arm on session 1 was termed "uncued1". Similarly, the times that each rat spent in the two types of arms on session 10 , after the eight pairing sessions, were termed "cued2" and "uncued2". Thus, it was possible to calculate a ratio score for each rat of cued1/cued $1+$ uncued 1 and compare this with cued2/cued $2+$ uncued 2 to assess the effect of pairing on preference for each rat. The data were log transformed before analysis because this is recommended when comparing proportional change (Hair et al., 1995). ANOVA was then performed using the factors "pairing" and "group". The effect of pairing was significant $\left[F_{(1,17)}=5.69\right.$;

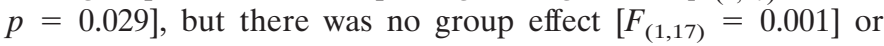
group $\times$ pairing interaction $\left[F_{(1,17)}=0.113\right]$.

\section{Entries to arms}

These data were analyzed using the same method of calculating ratio scores as the "time in arms" above. A significant effect of pairing was found $\left[F_{(1,17)}=7.29 ; p=0.015\right]$, but there was no group effect $\left[F_{(1,17)}=0.015\right]$ or group $\times$ pairing interaction $\left[F_{(1,17)}=0.023\right]$. Total entries to arms on session 1 only were also compared between the groups to assess the difference in overall levels of exploratory behavior (Fig. 4). Student's $t$ test performed on these data confirmed that the MD group made significantly more arm-entries than the SHAM group $\left[t_{(18)}=2.77 ; p=0.006\right)$.

\section{Open field}

There was no difference between the groups on line crossings $(t=$ $1.09 ;$ df $=18 ; p=0.15$; group means: $\mathrm{MD}=96.83$; $\mathrm{SHAM}=$ $75.30)$, or on inward line crossings $(t=0.88$; df $=18 ; p=0.20$; group means: $\mathrm{MD}=23.83$; SHAM $=18.20)$, or on time spent in inner segments $(t=0.20 ; \mathrm{df}=18 ; p=0.42$; group means: $\mathrm{MD}=$ 91.00; SHAM = 70.40).

\section{Matching to place}

\section{Acquisition of matching to place task}

Comparisons using the number of trials to the acquisition criterion (Fig. 5) showed that the MD group was significantly slower to learn the task [two-tailed Student's $t$ test: $t_{(18)}=3.25 ; p=0.004$; group means: $\mathrm{MD}=151.6 ; \mathrm{SHAM}=109.8]$. Errors to criterion (Fig. 5) revealed a similar acquisition difference between the two groups $\left[t_{(18)}=3.68 ; p=0.002\right.$; group means: $\mathrm{MD}=75.9$; SHAM $=54.1]$.

Both groups began the acquisition phase by performing well below chance levels (mean group correct trials over the first 30 trials: $\mathrm{MD}=5.1 ; \mathrm{SHAM}=6.6$; chance $=15)$. Two-tailed $t$ tests confirmed that both groups' performance at this stage was significantly below chance [MD: $t_{(9)}=9.71, p<0.001$; SHAM: $t_{(9)}=$ 13.3, $p<0.001]$. Furthermore, at this initial stage there was no difference in the performance levels of the two groups [first 30 trials, $t_{(18)}=0.98 ; p=0.34$, two-tailed].

To examine more closely the way that the two groups acquired the matching task, the acquisition process was divided into two phases. Very low scores, (3/12 or lower) were described as "perseveration" (probability of scoring 3/12 or lower $=0.073$ ), 
Figure 2. Photomicrograph of coronal sections (Nissl stain) showing the appearance of nucleus medialis dorsalis in a normal animal (left) and in the MD animal with the median-sized lesion (right). The photomicrograph shows not only the loss of neurons within nucleus medialis dorsalis but also the resultant contraction of the region. $H$, Hippocampus; $M D$, nucleus medialis dorsalis.
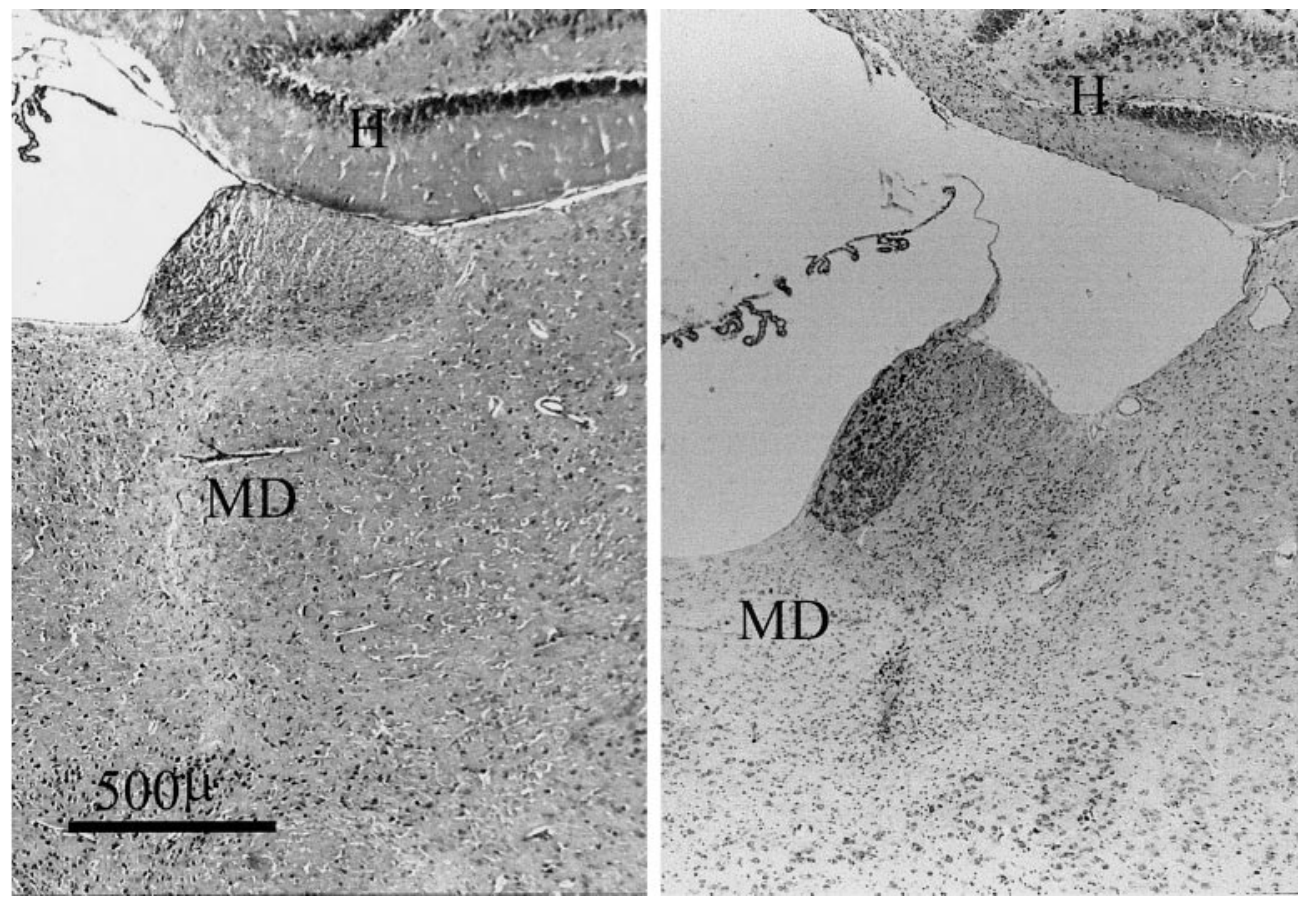

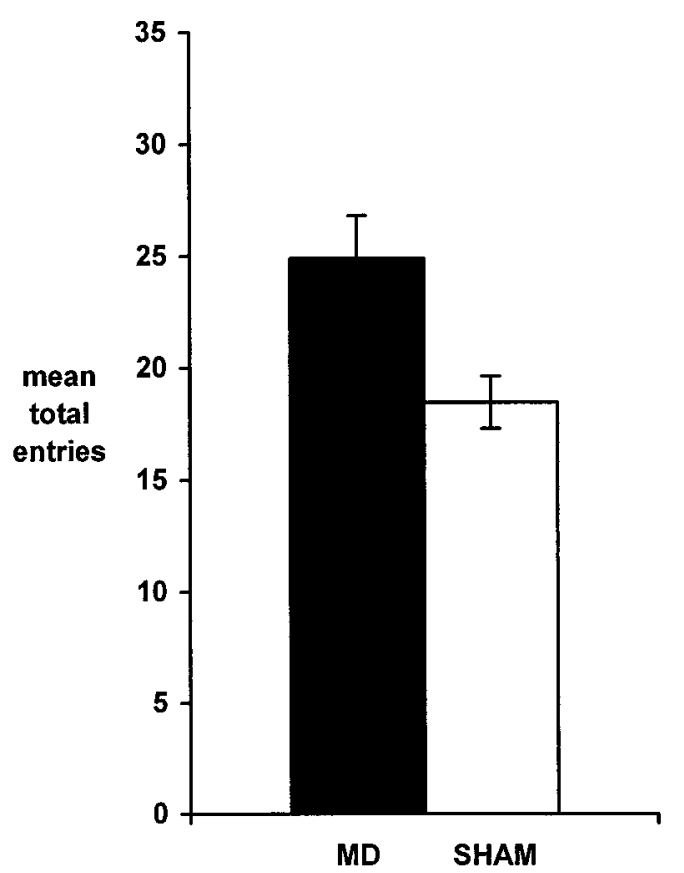

Figure 4. Conditioned cue preference: exploratory behavior as measured by entries into arms on the initial test session.

errors over these two phases, and ANOVA of the error data using the factors group and phase showed a significant effect of group $\left[F_{(1,18)}=5.74 ; p=0.028\right]$, but not phase $\left[F_{(1,18)}=2.88 ; p=0.107\right]$ or group by phase interaction $\left[F_{(1,18)}=1.31 ; p=0.267\right]$. Despite the lack of a significant interaction, analysis of the simple effects showed that the two groups differed on the perseveration measure $(p<0.05)$ but not on the learning measure. For purposes of comparison, the acquisition data are depicted in Figure 7 by blocks of trials only. Unlike the above method of analysis (Fig. 6), this more conventional method clearly fails to show the important difference in the way that the two groups of rats learned the task. trials up to the task acquisition criterion of five or more correct responses on five successive days. Figure 6 shows the pattern of whereas scores of 4/12 or higher were described as "learning". The perseveration scores were assumed to correspond to the rats' dich to solve the matching task by nonmatching. The made by each rat in a running window of 12 trials, beginning with trials $1-12$ and advancing the window by one trial at a time. The initial perseveration phase was deemed to have ended when the rat achieved a score of four or more correct responses in a window of 12 trials. The learning phase comprised all subsequent 
Trials to criterion

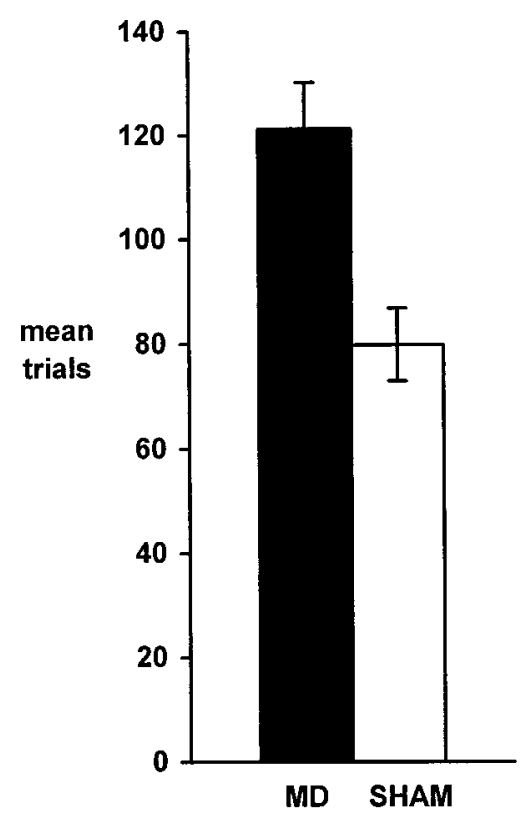

Perseveration

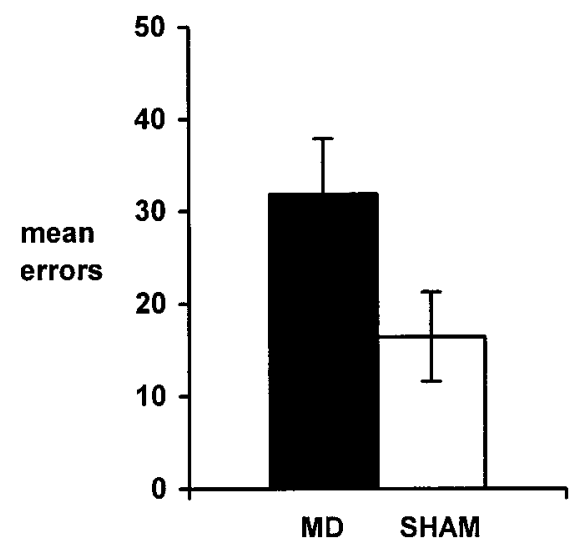

\section{Errors to criterion}

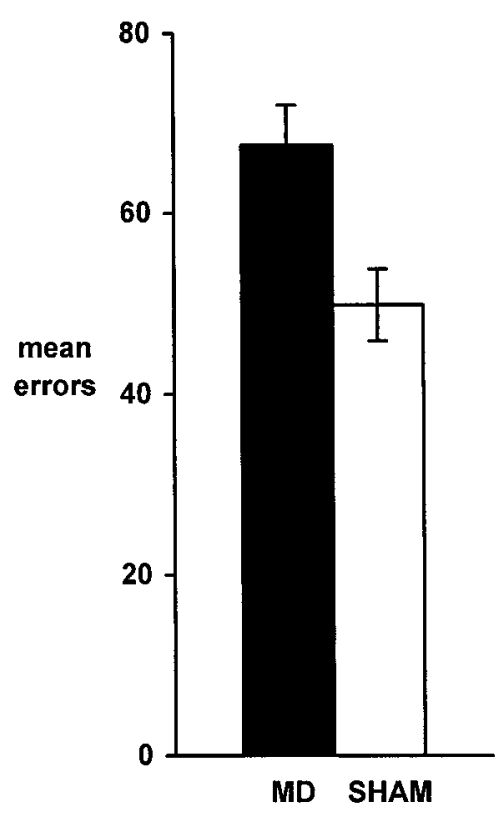

Figure 5. Acquisition of the T-maze matching to place task. Mean numbers of trials to criterion (left) and errors to criterion (right) are shown for both groups.

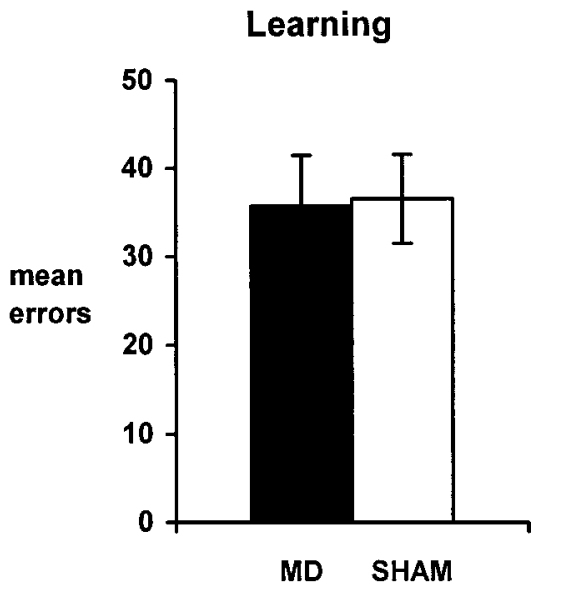

Figure 6. Acquisition of the T-maze matching to place task. The charts depict the mean number of errors made during the two acquisition phases of perseveration (rats performing below chance) and learning (rats performing at or above chance).

\section{Matching to place with delays}

The performance of the two groups over the three delay conditions is shown in Figure 8. ANOVA was performed using the factors of group and delay. There was a strong effect of delay $\left[F_{(2,36)}=14.05 ; p<0.001\right]$ but no effect of group $\left[F_{(1,18)}=0.93\right]$ or group by delay interaction $\left[F_{(2,36)}=0.66\right]$.

Normal matching to position

After the delay conditions each rat underwent three sessions of matching to sample with $10 \mathrm{sec}$ retention intervals (18 trials). Both groups performed the task at a high level (mean scores: $\mathrm{MD}=16$; $\mathrm{SHAM}=16.3$ ), and there was no evidence of a group difference.

\section{Reversal to nonmatching to place}

Overall acquisition of the nonmatching task by the two groups barely differed (group means of errors to criterion: $\mathrm{MD}=39.7$; $\mathrm{SEM}=2.45 ; \mathrm{SHAM}=38.0 ; \mathrm{SEM}=3.39$ ). However, analysis of the pattern of errors by two phases, performed in the same way as for the matching to place task, showed that the two groups differed in the way that they achieved acquisition (Fig. 9). ANOVA using the factors group and phase showed no effect of group $\left[F_{(1,18)}=0.05 ; p=0.82\right]$, or of phase $\left[F_{(1,18)}=0.01 ; p=\right.$ $0.915]$, but there was a significant group by phase interaction $\left[F_{(1,18)}=8.75 ; p=0.008\right]$. This interaction arose from the MD animals making more perseverative errors and fewer learning errors (Fig. 9).

\section{DISCUSSION}

The key finding in the present study was the deficit in learning to switch a response rule after neurotoxic damage to nucleus medialis dorsalis. The selective nature of the deficit helps to rule out other possible explanations and, at the same time, reveals a clear parallel with the effects of prefrontal damage. In view of the very dense, reciprocal connections between the prefrontal cortex and nucleus medialis dorsalis these results suggest that the perseverative deficits after prefrontal cortex and medial dorsal thalamic damage reflect dysfunctions in the same system.

In the present study, the T-maze tests revealed a consistent dissociation within the behavioral effects of lesions in nucleus 


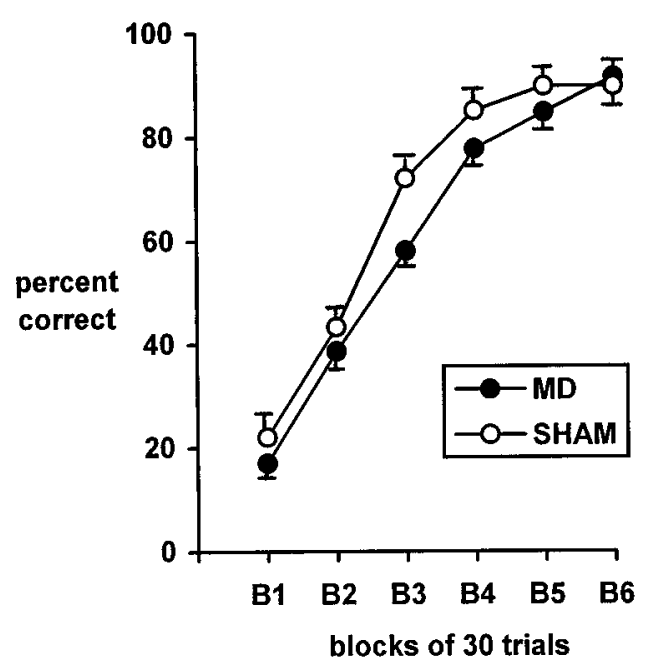

Figure 7. Acquisition of the T-maze matching to place task. The graph shows the pattern of acquisition over six blocks of 30 trials. Rats that acquired the task before block 6 were assumed to continue performing at the same level as when they reached the acquisition criterion.

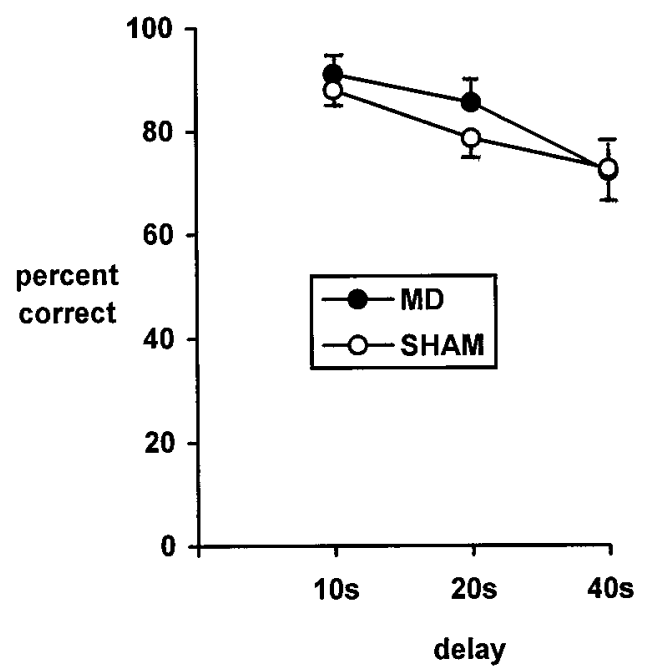

Figure 8. T-maze matching to place performance. The graph shows the percentage of correct trials (maximum 20) performed by the two groups over three delay conditions after acquisition.

medialis dorsalis. Thus, there was no evidence that the thalamic lesions disrupted the ability of the rats to distinguish which arm had been most recently visited (working memory), but the same rats were impaired at shifting from a preferred response rule. Evidence of their intact spatial working memory comes from the normal performance of the MD animals over retention delays (20 and $40 \mathrm{sec}$ ) that were of sufficient length to preclude possible ceiling effects. Furthermore, the rats with medialis dorsalis lesions persistently performed below chance at the outset of matching training, at a level that was comparable to that of the control rats. This unusually poor level of performance is to be expected as normal rats have a very strong, innate bias to alternate in the T-maze (Richman and Dember, 1986) i.e., to turn in the opposite direction to that rewarded in the matching condition. Thus, the highly significant performance below chance reflects the comparable ability of both sets of rats to remember the most recently visited arm.
This sparing of spatial working memory is consistent with recent studies that have helped to distinguish the contributions of other nuclei or tracts adjacent to medialis dorsalis. Although selective lesions of the dorsomedial thalamus, similar to those in the present task, have little or no effect on nonmatching to place tasks (Hunt and Aggleton, 1991, 1998; Kessler et al., 1982; Neave et al., 1993), bilateral damage to the anterior thalamic nuclei will produce severe, lasting deficits on the same tasks (Aggleton et al., 1995a, 1996). These deficits are still present when the lesions are placed in subfields of the anterior thalamic nuclei (Aggleton et al., 1996; Byatt and Dalrymple-Alford, 1996), highlighting the need to minimize encroachment into these nuclei. Likewise, there is evidence that cutting the mamillothalamic tract is sufficient to impair T-maze alternation (Thomas and Gash, 1985). Thus, although some studies have reported that lesions in the dorsomedial thalamic region can disrupt spatial working memory (Stokes and Best, 1988, 1990a,b,c), careful analysis indicates that in many of these instances the lesions have encroached rostrally to involve the anterior thalamic nuclei.

Despite their intact spatial working memory, the MD animals were impaired at acquiring the matching rule. One possible explanation is that the lesion has a general effect on the ability of rats to learn the reference memory component of a task, in this case, the rule to match. A general failure to learn task rules does not, however, seem likely because the same rats were readily able to learn the nonmatching to place task once they had ceased perseverating on the original rule (Fig. 9). Similarly, performance during the learning phase of the matching task appeared normal (Fig. 6). Also, previous studies have found that very similar thalamic lesions do not disrupt the ability to acquire the delayed nonmatching to position task in an automated chamber (Neave et al., 1993), nor do they affect the ability to learn the position of a platform in the Morris water maze (Kolb et al., 1982). In both instances, medial dorsalis lesions spared the learning of a response rule in a spatial task.

Another possible explanation for the deficit in learning the matching rule is that the lesions left the rats unresponsive to the food reinforcers. This account seems unlikely, however, given the ability of the MD rats to improve rapidly once they ceased perseverating (Figs. 6, 9). Furthermore, these same rats showed normal performance on the cue preference task. In this task, a salient visual cue (black or white surround) was associated with food reward, independent of spatial position. The two groups did not differ on either of the two preference measures (time in arms, number of entries to arms). This result contrasts with the findings of McAlonan et al. (1993) who found that ibotenic acid lesions in medialis dorsalis abolished the acquisition of conditioned cue preference. The sizes of the lesions in the two studies appear comparable, and the basic task procedures are similar. For this reason the difference in outcome is of interest but remains unresolved. Despite this, there is no evidence that the present MD rats failed the matching task because they were insensitive to the reinforcers or were unable to associate a reinforcer with a specific cue.

Having excluded these other possibilities, it can be concluded that lesions of nucleus medialis dorsalis lead to a selective deficit in the ability to switch from a preferred strategy to a new strategy. The initial acquisition deficit arose from a failure to switch from an innately preferred strategy (nonmatching) to a new strategy (matching), as reflected by a specific increase in perseverative errors. Similarly, the abnormal pattern of errors in the subsequent reversal to a nonmatching rule also arose from an excess of 
Perseveration

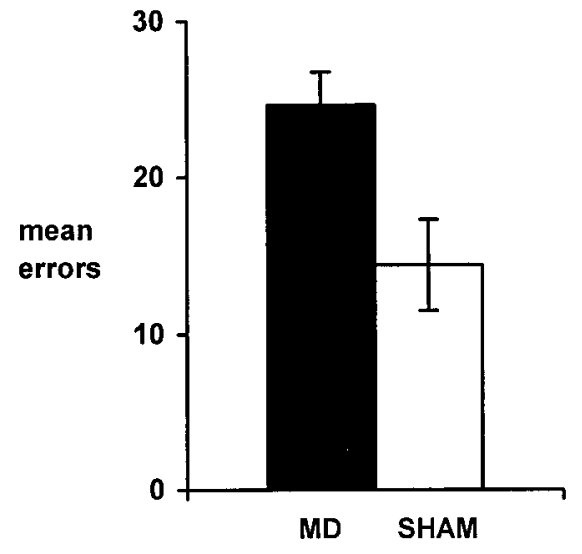

\section{Learning}

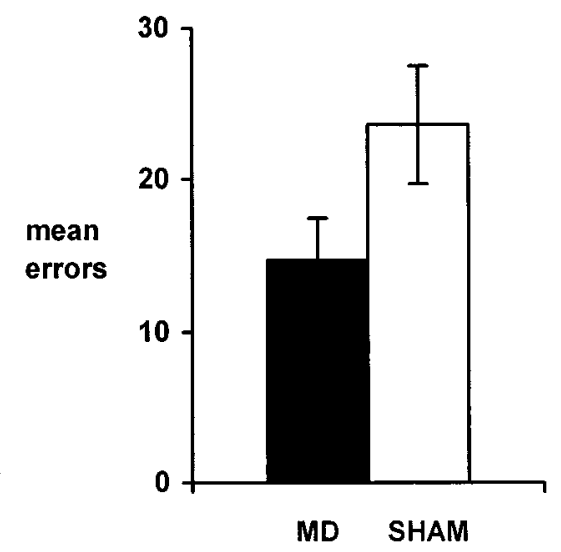

Figure 9. Acquisition of the reversal to T-maze nonmatching to sample task. The mean group error scores for the two groups have been divided into two phases: $\mathrm{P}=$ perseveration phase in which rats are performing below chance; $\mathrm{L}=$ learning phase in which rats are performing at or above chance. perseverative errors. It is most unlikely that these failures arose from an inability to shift attention to the critical stimulus dimension (Dias et al., 1996) because the matching rule uses the same class of stimuli as the preferred, nonmatching rule. Consequently, the deficit can be better characterized as a failure to shift response rules. This would also explain why lesions of nucleus medialis dorsalis have little or no impact on the standard radial arm maze procedure, because the task accords with natural foraging strategies (Hunt and Aggleton, 1998). In contrast, deficits are found when the procedure is modified so that the selection of some arms is never rewarded (Hunt and Aggleton, 1998), because the rat now has to withhold the normal foraging strategy of visiting all arms.

Nucleus medialis dorsalis has dense, reciprocal connections with prefrontal cortex, and it is, therefore, of great interest that damage to the prefrontal cortex also results in perseverative deficits (Mishkin, 1964; Kolb, 1984). Furthermore, frontal damage can result in hyperactivity (Kolb, 1984; Kolb et al., 1982), and evidence of hyperactivity was found in the present MD group. On the very first cue preference session, the rats with thalamic lesions made a greater number of arm entries than the control rats, indicating that these rats were less inhibited in exploring the test apparatus (Hunt and Aggleton, 1998). Interestingly, McAlonan et al. (1993) also reported evidence of increased activity during the conditioned cue preference task. Although there was no evidence that the MD rats were hyperactive in the open field test in the present study, other studies have reported an increase in exploration in a variety of test conditions after lesions of the dorsomedial thalamus (Kolb et al., 1982; Kolb, 1984; Beracochea et al., 1989; Hunt and Aggleton, 1998).

Perhaps the most important aspect of the present study is the way that the pattern of deficits found after lesions of nucleus medialis dorsalis echo those seen after selective lesions of the prefrontal cortex and, in particular, the prelimbic cortex of the rat. Neurotoxic lesions in both sites can spare tests of allocentric spatial working memory as long as the task rules accord with the innate strategies on the animal. Thus, selective damage in the medial prefrontal cortex has either transient or no disruptive effects on standard tests of spatial memory (Shaw and Aggleton, 1993; Aggleton et al., 1995b) i.e., like lesions of the medial dorsal nucleus it did not produce a working memory deficit per se. More robust deficits do, however, appear when rats are required to change response rules. Examples of this are found in studies looking at place reversals in the water maze (De Bruin et al.,
1994) and in shifting from standard to modified versions of the radial arm maze (Kolb et al., 1982; Seamans et al., 1995; Delatour and Gisquet-Verrier, 1996). Of special relevance is the finding that selective neurotoxic lesions of the prelimbic cortex can spare delayed nonmatching to place in a T-maze (forced alternation) but impair delayed matching to place (Dias and Aggleton, 1997). This pattern of results, which directly corresponds to that found in the present study, indicates that the connections between the nucleus medialis dorsalis and the prelimbic cortex are important for the ability to shift response rules. Because lesions of medialis dorsalis do not increase response bias on tests such as delayed nonmatching to position (Neave et al., 1993) this combined prefrontal-thalamic involvement may be especially important for innate or central set preferences (Mishkin, 1964).

\section{REFERENCES}

Aggleton JP, Neave N, Nagle S, Hunt PR (1995a) A comparison of the effects of anterior thalamic, mamillary body, and fornix lesions on reinforced spatial alternation. Behav Brain Res 68:91-101.

Aggleton JP, Neave N, Nagle S, Sahgal A (1995b) A comparison of the effects of medial prefrontal, cingulate cortex, cingulum bundle lesions on tests of spatial memory: evidence of a double dissociation between frontal and cingulum bundle contributions. J Neurosci 15:7270-7281.

Aggleton JP, Hunt PR, Nagle S, Neave N (1996) The effects of selective lesions within the anterior thalamic nuclei on spatial memory in the rat. Behav Brain Res 81:189-198.

Beracochea DJ, Jaffard R, Jarrard L (1989) Effects of anterior or dorsomedial thalamic ibotenic lesions on learning and memory in rats. Behav Neural Biol 51:364-376.

Byatt G, Dalrymple-Alford JC (1996) Both anteromedial and antero ventral thalamic lesions impair radial-maze learning in rats. Behav Neurosci 110:1335-1348.

Cohen JD, Braver TS, O'Reilly RC (1996) A computational approach to prefrontal cortex, cognitive control, and schizophrenia: recent developments and current challenges. Philos Trans R Soc Lond B Biol Sci 351:1515-1527.

de Bruin JP, Sanchez-Santed F, Heinsbroek RP, Donker A, Postmes P (1994) A behavioral analysis of rats with damage to the medial prefrontal cortex using the Morris water maze: evidence for behavioral flexibility, but not for impaired spatial navigation. Brain Res 652: 323-333.

Delatour B, Gisquet-Verrier P (1996) Prelimbic cortex specific lesions disrupt delayed-variable response tasks in the rat. Behav Neurosci 110:1282-1298.

Dias R, Aggleton JP (1997) A comparison of the effects of prelimbic and anterior cingulate cortex lesions in the rat. Soc Neurosci Abstr 23:1606.

Dias R, Robbins TW, Roberts AC (1996) Dissociation in prefrontal cortex of affective and attentional shifts. Nature 380:69-72. 
Hair JF, Anderson RE, Tatham RL, Black WC (1995) Multivariate data analysis with readings, Ed 5, Englewood Cliffs, NJ: Prentice Hall.

Hunt PR, Aggleton JP (1991) Medial dorsal thalamic lesions and working memory in the rat. Behav Neural Biol 55:227-246.

Hunt PR, Aggleton JP (1998) Analysing the nature of the spatial working memory deficit following neurotoxic medial dorsal thalamic lesions in rats. Behav Brain Res, in press.

Keppel G (1973) Design and analysis: a researcher's handbook. Englewoods Cliffs, NJ: Prentice Hall.

Kessler J, Markowitsch HJ, Otto B (1982) Subtle but distinct impairments of rats with chemical lesions of the thalamic mediodorsal nucleus, tested in a radial arm maze. J Comp Physiol Psychol 96:712-720.

Kolb B (1984) Functions of the frontal cortex of the rat: a comparative review. Brain Res Rev 8:65-98.

Kolb B, Nonneman AJ, Singh RK (1974) Double dissociation of spatial impairments and perseveration following selective prefrontal lesions in rats. J Comp Physiol Psychol 87:772-780.

Kolb B, Pittman K, Sutherland RJ, Whishaw IQ (1982) Dissociation of the contributions of the prefrontal cortex and dorsomedial thalamic nucleus to spatially guided behavior in the rat. Behav Brain Res 6:365-378.

Markowitsch HJ (1982) Thalamic mediodorsal nucleus and memory: a critical evaluation of studies in animals and man. Neurosci Biobehav Rev 6:351-380.

McAlonan GM, Robbins TW, Everitt BJ (1993) Effects of mediodorsal thalamic and ventral pallidal lesions on the acquisition of a conditioned place preference: further evidence for the involvement of the ventral striatopallidal system in reward related processes. J Neurosci 52:605-620.

Mishkin M (1964) Perseveration of central sets after frontal lesions in man. In: The frontal granular cortex and behavior (Warren JM, Akert K, eds), pp 219-294. New York: McGraw-Hill.

Neave N, Sahgal A, Aggleton JP (1993) Lack of effect of dorsomedial thalamic lesions on automated tests of spatial memory in the rat. Behav Brain Res 55:39-49.

Pellegrino LJ, Cushman AJ (1967) Stereotaxic atlas of the rat brain. New York: Appleton-Century-Crofts.

Richman CL, Dember WN (1986) Spontaneous alternation behavior in animals: a review. Current Psychol Res and Rev 5:358-391.

Robbins TW (1996) Dissociating executive functions of the prefrontal cortex. Philos Trans R Soc Lond B Biol Sci 351:1463-1470.

Sahgal A (1993) Practical behavioural neuroscience: problems, pitfalls, and suggestions In: Behavioural neuroscience: a practical approach, Vol I (Sahgal A, ed), pp 1-8. Oxford: Oxford UP.

Seamans JK, Floresco SB, Philips AG (1995) Functional differences between the prelimbic and anterior cingulate regions of the rat prefrontal cortex. Behav Neurosci 6:1063-1073.

Shaw C, Aggleton JP (1993) The effects of fornix and medial prefrontal lesions on delayed non-matching to sample by rats. Behav Brain Res 54:91-102.

Stokes KA, Best PJ (1988) Mediodorsal thalamic lesions impair radial maze performance in the rat. Behav Neurosci 102:294-300.

Stokes KA, Best PJ (1990a) Mediodorsal thalamic lesions impair "reference" and "working" memory in rats. Physiol Behav 47:471-476.

Stokes KA, Best PJ (1990b) Medial dorsal thalamus lesions in rats impair radial-arm maze performance in a cued environment. Psychobiology 18:63-67.

Stokes KA, Best PJ (1990c) Response biases do not underlie the radial maze deficit in rats with mediodorsal thalamus lesions. Behav Neural Biol 53:334-345.

Thomas GJ, Gash DM (1985) Mamillothalamic tracts and representational memory. Behav Neurosci 99:621-630.

Van der Kooy D (1985) Place conditioning: a simple and effective method for assessing the properties of drugs In: Methods of assessing the motivational properties of abused drugs (Bozarth M, ed), pp 229240. New York: Springer. 